\title{
Projet de Recherche sur le Désir Sexuel
}

\section{dans l'Hypogonadisme Masculin}

L'équipe de recherche «Psychobiologie de la Sexualité et de la Reproduction Humaines» animée par le Docteur Serge Stoléru (Unité 292, INSERM) et Jérôme Redouté, Doctorant, recherche la collaboration de partenaires cliniciens en vue d'une étude du Désir Sexuel dans l'Hypogonadisme Masculin.

L'objectif de cette recherche est d'étudier la baisse du désir sexuel dans l'hypogonadisme et les troubles corrélatifs de l'activation des régions cérébrales dont le fonctionnement est associé au désir sexuel.

Dans un premier temps, notre équipe a, en effet, montré, à l'aide de la tomographie par émission de positions, que certaines régions cérébrales avaient une activité corré1ée au désir et à l'excitation sexuels : ce sont notamment le cortex cingulaire gauche, la région insulaire droite, la tête du noyau caudé droit et le gyrus orbitaire droit. Nous cherchons maintenant à déterminer si l'activation de ces régions est différente chez les hommes présentant une diminution du désir sexuel dans le contexte d'un hypogonadisme.

Connaissant les fonctions respectives de ces régions cérébrales, les résultats de cette recherche nous permettront notamment de mieux déterminer si l'activation de ces régions est diminuée lorsque le taux de la testostérone plasmatique est abaissé et de mieux comprendre les relations entre testostérone, cerveau et désir sexuel.
Les critères de recrutement essentiels de l'étude sont les suivants :

testostérone $<150 \mathrm{mg} / 100 \mathrm{ml}$ en l'absence d'androgénothérapie, pas d'anomalie du caryotype (tel qu'un syndrome de Klinefelter), sexe masculin, pas de toxicomanie, orientation exclusivement hétérosexuelle, âge entre 20 et 50 ans, sujets droitiers.

Si vous souhaitez faire partie d'un groupe de cliniciens intéressés par ce projet de recherche, veuillez, SVP, contacter le

\section{Docteur S. Stoléru,} U292 INSERM, CHU Bicêtre, Porte 26, 82 rue du Général Leclerc, 94276 Le Kemlin-Bicêtre Cedex;

Tél. 01.45.21.23.33; Fax: 01.45.21.20.75; e-mail: stoleru@lorelei.vjf.inserm.fr 